\title{
Synthesis of peptide-based nanoparticles on electrostatic complexation via microfluidic route.
}

\section{Luana J. Cattan*, Thays F. Naves, Naiara Godoi, Lucimara G. De la Torre.}

\begin{abstract}
The introduction of the naked DNA through the negatively charged cell membrane is an obstacle in the gene therapy field. Therefore, the eletrostatic condesation of the DNA by cationic polypeptides, such as polylysine (PLL), resultes in a posetively charged complex, facilitating its entrance on the cellular enviroment. Aiming to overcome the toxicity of PLL, it is complexed with anionic peptides, such as polyglutamic acid (PGA). Thus, this research project investigated the synthesis of the PLL/PGA/DNA ternary complex in bulk and microfluidics systems for further applications gene therapy.
\end{abstract}

Key words: Polypeptides, gene therapy, microfluidics.

\section{Introduction}

One of the nanotechnology targets is the production of nanoscale structures with application in gene therapy. In this context, emerges the interest in cationic polypeptides, such as polylysine (PLL), capable of condensing the naked DNA through electrostatic interaction, resulting in cationic complexes and facilitating the introduction of the genetic material through the anionic cell membrane. However, the complexation of PLL by anionic peptides, such as polyglutamic acid (PGA), aims to shield PLL's positive charge, reducing its citotoxicity.

Although there are studies reporting the synthesis of PLL/DNA complex in bulk process, microfluidics appears as a technology that allows greater control over the diffusion mechanism of the nanoparticles produced ${ }^{1}$. Thus, the synthesis of PLL/PGA/DNA ternary complex is investigated in both systems, in order to better understand the electrostatic complexation process for future applications in gene therapy.

\section{Results and Discussion}

In order to understand the behavior and the best conditions of the electrostatic association between PLL and DNA and PLL and PGA, the firsts bulk tests were performed by adding DNA to the PLL solution, parallel to the addition of PGA to the PLL solution, both complexes in different mass ratio, RPLL/DNA and RPLL/PGA respectively, from 0.5 to $50(\mathrm{~m} / \mathrm{m})$.

The PLL/DNA complexes showed in RPLL/DNA $=1$ low polydispersity (PDI) of 0.3 , mean diameter of $50 \mathrm{~nm}$ and positive zeta potential of $36 \mathrm{mV}$. Performing the same analysis for PLL/PGA, the RPLL/PGA=10 showed PDI of 0.3 , mean diameter of $70 \mathrm{~nm}$ and positive zeta of $30 \mathrm{mV}$. Considering those satisfactory results, further bulk tests were carried out, producing the ternary complex PLL/PGA/DNA according to the flowchart:

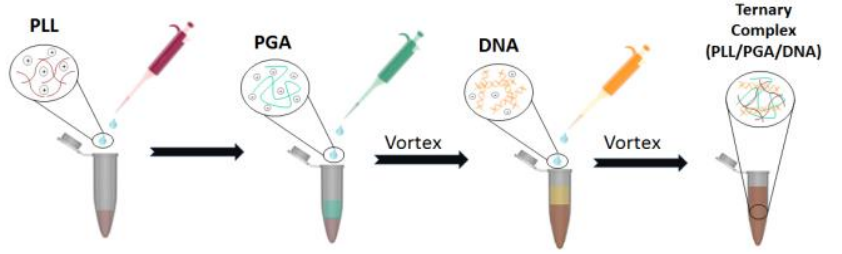

Image 1. Schematic diagram of the synthesis of complex PLL/PGA/DNA in batch conventional process by pipetting.

Different reaction orders were tested, starting by the PLL/PGA complex, in the mass ratio RPLL/PGA $=10$, and further addition of DNA, varying the mass ratio RPLL/DNA from 0.5 to $20(\mathrm{~m} / \mathrm{m})$. Similarly, we varied the addition ratio of PGA to the PLL/DNA complex, in mass ratio RPLL/DNA $=1$, and obtained the following results:

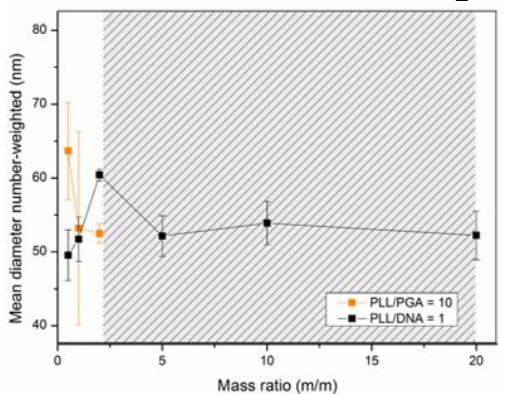

Image 2: Ternary complex PLL/PGA/DNA characterized in terms of mean diameter number-weighted. Mass ratio hatched correspond to data not agreed to the equipment quality report.

The results indicated that better synthesis condictions were achieved in the addition of PGA to the PLL/DNA complex, due to the larger scale of viable ratios and better physicalchemical proprieties. Thus, as the lower mean diameter, PDI of 0.3 and positive zeta of $40 \mathrm{mV}$ in was observed in RPLL/PGA $=5$, this complex ratio was synthesized in microfluidic device, resulting in a nanoparticle with mean diameter of $87.75 \mathrm{~nm}, \mathrm{PDI}$ of 0.4 and positive zeta potential of $38.9 \mathrm{mV}$.

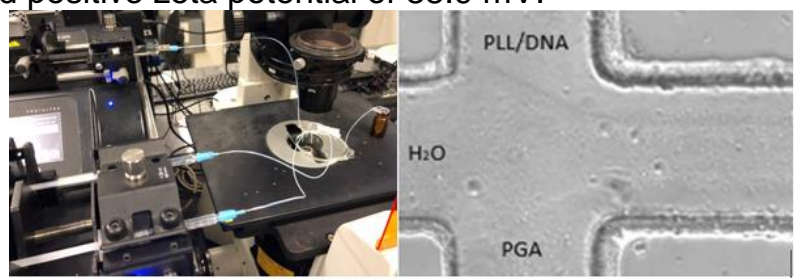

Image 3: Production of ternary complex in microfluidic device with a diffusive central aqueous flow barrier and total flow rate of $30 \mu \mathrm{L} / \mathrm{min}$.

Despite the similar results obtained in both synthesis methotds, the microfluidics approach deepens the knowledge over the eletrostatic complexation mechanism.

\section{Conclusions}

The results obtained in microfluidics platform reveal its potential on the exploration of different synthesis conditions in order to improve the production of polypeptide-based nanoparticles. Further biological tests should be carried out to asses its potential application in gene therapy.

${ }^{1}$ WHITESIDES, G. M. The origins and the future of microfluidics. Nature, $v$ 442, n. 7101, p. 368-73, 2006. 